\title{
Epigenetic inactivation of FAT4 contributes to gastric field cancerization
}

\author{
Satoshi Yoshida ${ }^{1,3} \cdot$ Satoshi Yamashita $^{1} \cdot$ Tohru Niwa $^{1} \cdot$ Akiko Mori $^{1} \cdot$ \\ Seiji Ito $^{2} \cdot$ Masao Ichinose ${ }^{3} \cdot$ Toshikazu Ushijima ${ }^{1}$
}

Received: 21 June 2015/Accepted: 5 January 2016/Published online: 20 January 2016

(c) The International Gastric Cancer Association and The Japanese Gastric Cancer Association 2016

\begin{abstract}
Background Gastric cancer (GC) is highly influenced by aberrant methylation, and accumulation of aberrant methylation in gastric mucosae produces an epigenetic field for cancerization. Nevertheless, the individual driver genes involved in such field cancerization are still unclear. Here, we aimed to demonstrate that FAT4, a novel tumor suppressor identified by exome sequencing of $\mathrm{GC}$, is methylation-silenced and that such methylation is involved in epigenetic field cancerization for GC.

Methods A transcription start site was determined by the $5^{\prime}$ rapid amplification of complementary DNA ends method. DNA methylation was analyzed by bisulfite sequencing with use of a next-generation sequencer or quantitative methylation-specific PCR. Gene expression was analyzed by quantitative reverse transcription PCR.

Results A single transcription start site was identified for FAT4 in gastric epithelial cells, and a CpG island was located in the FAT4 promoter region. FAT4 was highly methylated in two of $13 \mathrm{GC}$ cell lines and was not expressed in them. Removal of FAT4 methylation by a DNA demethylating agent (5-aza-2'-deoxycytidine) restored its expression in the two cell lines. In primary GC
\end{abstract}

Electronic supplementary material The online version of this article (doi:10.1007/s10120-016-0593-5) contains supplementary material, which is available to authorized users.

Toshikazu Ushijima

tushijim@ncc.go.jp

1 Division of Epigenomics, National Cancer Center Research Institute, 5-1-1 Tsukiji, Chuo-ku, Tokyo 104-0045, Japan

2 Department of Gastroenterological Surgery, Aichi Cancer Center Central Hospital, Nagoya, Japan

3 Second Department of Internal Medicine, Wakayama Medical University, Wakayama, Japan samples, FAT4 was methylated in 12 of 82 GCs (14.6\%). FAT4 methylation was associated with the presence of the $\mathrm{CpG}$ island methylator phenotype but not with prognosis, tumor invasion, lymph node metastasis, or histological types. In noncancerous gastric mucosae, high FAT4 methylation levels were associated with the presence of GC and Helicobacter pylori infection.

Conclusions FAT4 was methylation-silenced in GCs. Its methylation in gastric mucosae was associated with $H$. pylori infection and likely contributed to epigenetic field cancerization.

Keywords FAT4 - DNA methylation - Gastric cancer . $\mathrm{CpG}$ island methylator phenotype $\cdot$ Helicobacter pylori

$\begin{array}{ll}\text { Abbreviations } & \\ \text { 5-aza-dC } & \text { 5-Aza-2'-deoxycytidine } \\ \text { CGI } & \text { CpG island } \\ \text { CIMP } & \text { CpG island methylator phenotype } \\ \text { DBTSS } & \text { Database of Transcriptional Start Sites } \\ \text { GC } & \text { Gastric cancer } \\ \text { M-MSP } & \text { Primers specific to the methylated } \\ \text { primers } & \text { sequence } \\ \text { PMR } & \text { Percentage of methylated reference } \\ \text { qMSP } & \text { Quantitative methylation-specific PCR } \\ \text { RACE } & \text { Rapid amplification of complementary } \\ & \text { DNA ends } \\ \text { TSS } & \text { Transcription start site } \\ \text { Uni-MSP } & \text { Primer common to methylated and } \\ \text { primer } & \text { unmethylated DNA sequences }\end{array}$

\section{Introduction}

Genetic and epigenetic alterations are deeply involved in human cancers [1,2]. Notably, in gastric cancers (GCs), tumor suppressor genes, such as $\mathrm{CDHI}$ (which encodes 
E-cadherin), CDKN2A (which encodes p16), and MLH1 (which encodes DNA mismatch repair protein mutL homologue 1), are inactivated more frequently by epigenetic alterations-namely, aberrant DNA methylation of their promoter $\mathrm{CpG}$ islands (CGIs) - than by genetic alterations [3]. Also, in our recent comprehensive study on genetic and epigenetic alterations in GCs, activation of the Wnt pathway, inactivation of cell cycle regulation, and impairment of mismatch repair were more frequently caused by aberrant DNA methylation than by genetic alterations [4]. Aberrant DNA methylation is considered to be deeply involved in GC because Helicobacter pylori infection induces aberrant DNA methylation, which accumulates in noncancerous gastric tissues [5-9].

DNA methylation in gastric mucosae accumulates to higher levels in patients with GC than in healthy individuals [5-7], and in patients with multiple GCs than in those with a single GC $[10,11]$. These facts show that gastric tissue with an accumulation of aberrant DNA methylation is predisposed to cancer development, forming an epigenetic field for cancerization [12]. A multicenter prospective clinical study demonstrated the usefulness of measuring the severity of an epigenetic field in cancer risk diagnosis [13]. Mechanistically, in an epigenetic field, various but specific genes are methylated, including both driver and passenger genes [14, 15]. In addition to well-established tumor-suppressor genes, such as $C D H 1, C D K N 2 A$, and $M L H 1$, we recently showed that components of the SWI/SNF chromatin remodeler are also methylation-silenced as drivers in gastric mucosae [16]. However, an overall picture of driver genes involved in an epigenetic field is still unclear.

As a candidate driver involved in an epigenetic field in gastric mucosae, we focus here on FAT4 (which encodes FAT atypical cadherin 4, also known as cadherin family member 14, FAT-J, and cadherin-related family member 11 ), which was recently identified as a novel driver gene in GCs by exome analysis [17]. FAT4 was mutated $(5 \%)$ or deleted (4\%) in GCs, and small interfering RNA repression of FAT4 decreased cellular adhesion and increased migration and invasion of GC cells. Moreover, methylation of FAT4 was noted in breast and lung cancers [18, 19]. Biologically, FAT4 is one of the four human homologues of Drosophila fat, which is a member of the cadherin superfamily of genes and is involved in the planar cell polarity pathway $[20,21]$. FAT4 inactivation is associated with inactivation of the Hippo signaling pathway, which controls organ sizes [18, 22].

In this study, we aimed to demonstrate that FAT4 can be methylation-silenced and that its methylation contributes to epigenetic field cancerization in GCs.

\section{Materials and methods}

\section{Cell lines}

The AGS cell line was obtained from American Type Culture Collection (Manassas, VA, USA), and six other GC cell lines (KatoIII, MKN7, MKN28, MKN45, MKN74, and NUGC3) were obtained from the Japanese Collection of Research Bioresources (Tokyo, Japan). The GC2 cell line was a gift from M. Tatematsu (Aichi Cancer Center Research Institute, Nagoya, Japan). The 44As3, HSC39, HSC44, and HSC57 cells lines were gifts from K. Yanagihara (National Cancer Center Research Institute, Tokyo, Japan). The TMK1 cell line was a gift from W. Yasui (Hiroshima University, Hiroshima, Japan). The normal human gastric epithelial cell line HGEC6B was a gift from T. Kiyono (National Cancer Center Research Institute). Genomic DNA was extracted by the phenolchloroform method. RNA was extracted with ISOGEN (Nippon Gene, Tokyo, Japan).

\section{Tissue samples and clinicopathology information}

A normal gastric mucosa, used as a control in the methylation and expression analyses of GC cell lines, was obtained from a nontumorous region of a gastrointestinal stromal tumor surgical specimen from a 79-year-old man. Normal gastric mucosae used for the analysis of methylation levels induced by $H$. pylori were obtained from 33 healthy volunteers (17 with $\mathrm{H}$. pylori infection and 16 without) by endoscopic biopsy from 2004 to 2009. Noncancerous gastric mucosae were also obtained from 44 GC patients (28 with $H$. pylori infection and 16 without) by endoscopic biopsy from 2004 to 2010. H. pylori infection status was determined by a serum anti-H. pylori $\operatorname{IgG}$ antibody test (SRL, Tokyo, Japan). The degree of gastric atrophy was endoscopically diagnosed according to the Kimura-Takemoto classification [23]. On the basis of this classification, we defined classes $\mathrm{C}-1$ and $\mathrm{C}-2$ as a mild atrophy group, classes $\mathrm{C}-3$ and $\mathrm{O}-1$ as a moderate atrophy group, and classes O-2 and O-3 as a marked atrophy group.

GC tissues were obtained from surgical specimens of 82 GC patients (50 male and 32 female patients; average age 55.6 years, age range 29-69 years) who underwent gastrectomy because of GCs from 1995 to 2002. All GCs were histologically diagnosed according to the Japanese classification of gastric carcinoma and classified according to the Lauren classification system [24]; 69 diffuse-type and 13 intestinal-type GCs were included. All of the materials were collected after written informed consent had been obtained from the patients and approval had been obtained from the institutional review board. Genomic DNA and 


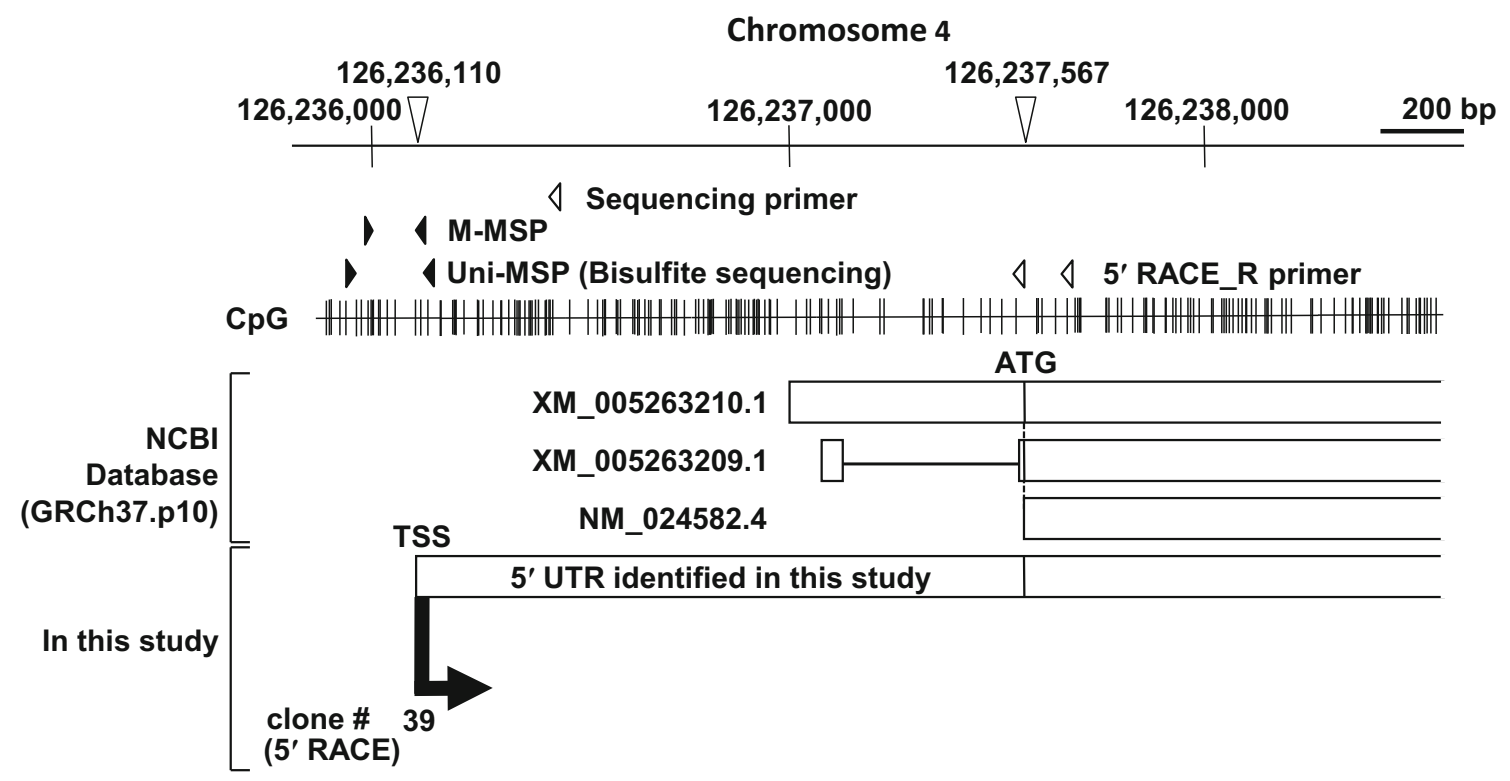

Fig. 1 Genomic structure around the FAT4 transcription start site (TSS). The TSS of human FAT4 in the National Center for Biotechnology Information (NCBI) database is located on chromosome 4 (NC_000004.11, 126,237,567). In contrast, the TSS in the Database of Transcriptional Start Sites (DBTSS) was located approximately $1,500 \mathrm{bp}$ upstream of the TSS registered in the NCBI database. Therefore, we performed $5^{\prime}$ rapid amplification of complementary DNA ends (RACE) using RNA from a gastric epithelial cell

total RNA were isolated from these samples in the same manner as from the cell lines.

\section{$5^{\prime}$ rapid amplification of complementary DNA ends}

$5^{\prime}$ rapid amplification of complementary DNA ends (RACE) was performed with a GeneRacer kit (Invitrogen, Carlsbad, CA, USA) and RNA from a normal human gastric epithelial cell line, HGEC6B. The PCR product was cloned into a pGEM-T Easy vector (Promega, Madison, WI, USA), and 39 clones were sequenced because a single PCR product was obtained. Sequencing was performed with a sequencing primer about 1,100 bp upstream of the $5^{\prime}$ RACE primer (Fig. 1, Table S1).

\section{5-Aza-2'-deoxycytidine and/or trichostatin A treatment}

AGS and GC2 cells were seeded at a density of $3 \times 10^{5}$ cells per $10-\mathrm{cm}$ plate on day 0 . For 5 -aza-2'-deoxycytidine (5-aza-dC; Sigma, St Louis, MO, USA) treatment, cells were exposed to a medium containing 1 or $3 \mu \mathrm{M} 5$-aza-dC (dissolved in phosphate-buffered saline) on days 1 and 3 and harvested on day 5. For a treatment with 5-aza-dC and trichostatin A (Sigma), the cells were additionally exposed to a medium containing $0.3 \mu \mathrm{M}$ trichostatin A on day 4 . line and identified a single TSS in cells from the stomach. Although two transcript variants of FAT4 are registered in the NCBI database, they were not detected in the $5^{\prime}$ RACE experiment here. Arrow TSS, arrowheads positions of primers for sequencing in $5^{\prime}$ RACE, methylation-specific PCR, and bisulfite sequencing, $M-M S P$ primers specific to methylated DNA, Uni-MSP primers common to methylated and unmethylated DNA sequences, UTR untranslated region

Mock-treated cells exposed to a medium containing only phosphate-buffered saline were used as a negative control.

\section{Sodium bisulfite modification and bisulfite sequencing}

Bisulfite modification was performed with $1 \mu \mathrm{g}$ of BamHIdigested genomic DNA as previously described [25], and the modified DNA was suspended in $40 \mu \mathrm{l}$ of TE buffer. To use next-generation sequencing technology, which allows the sequencing of a large number of DNA molecules, amplicons were obtained by PCR performed with primers common to methylated and unmethylated DNA sequences (Uni-MSP primers; Table S2) and $1 \mu \mathrm{L}$ of the sodium bisulfite treated DNA. From 100 ng of amplicons purified by a Zymo-Spin I column (Zymo Research, Irvine, CA, USA), a DNA library was prepared with an Ion fragment library kit (Life Technologies, Carlsbad, CA, USA) and an Ion Xpress barcode adaptors 1-96 kit (Life Technologies). Multiple libraries were pooled after unique barcoding and mixed with Ion Spheres, and emulsion PCR was conducted with an Ion OneTouch 2 (Life Technologies) with an Ion PGM template OT2 400 kit (Life Technologies). The emulsion PCR product, a complex of Ion Spheres and amplified DNA, was washed, concentrated with use of an Ion OneTouch ES enrichment module (Life Technologies), 
and loaded onto an Ion 314 chip (Life Technologies). Sequencing was conducted with an Ion PGM sequencer (Life Technologies) with an Ion PGM sequencing $400 \mathrm{Kit}$ (Life Technologies). The sequences obtained were converted to $\mathrm{CpG}$ methylation statuses by use of a plug-in for ION Suite based on Bismark (Babraham Bioinformatics, Babraham, UK). On the basis of the numbers of methylated and unmethylatedmolecules, a methylation level was calculated as the fraction of methylated molecules in the total number of DNA molecules (methylated molecules plus unmethylated molecules). To use conventional sequencing technology, PCR was also conducted with Uni-MSP primers. After the PCR product had been cloned into the pGEM-T Easy vector, 15 to 16 clones were sequenced.

\section{Quantitative methylation-specific PCR}

For quantitative methylation-specific PCR (qMSP), methylated DNA was amplified with primers specific to the methylated sequence (M-MSP primers), and both methylated and unmethylated DNA were amplified with UniMSP primers (Fig. 1). Real-time PCR was conducted with SYBR Green I (BioWhittaker Molecular Applications, Rockland, ME, USA), a CFX Connect ${ }^{\mathrm{TM}}$ real-time PCR system (Bio-Rad Laboratories, Hercules, CA, USA), and 1 $\mu \mathrm{L}$ of the sodium bisulfite treated DNA. Temperatures that allow specific annealing in the presence of SYBR Green I were determined with fully methylated and unmethylated DNA. The fully unmethylated DNA was prepared by amplification of the genomic DNA of healthy male peripheral blood cells twice with a GenomiPhi DNA amplification kit (GE Healthcare Bio-sciences, Little Chalfont, UK). The fully methylated DNA was prepared by methylation of the fully unmethylated DNA with SssI methylase (New England Biolabs, Beverly, MA, USA). The primers and PCR conditions are given in Table S2. Standard samples with known numbers of template DNA molecules were prepared by serial dilution of a purified PCR product. The methylation level was calculated as the fraction of methylated molecules in the total DNA molecules. The percentage of methylated reference (PMR) was calculated as the fraction of the methylated reference [(methylated molecules in a sample)/(total DNA molecules in a sample) $] /[$ (methylated molecules in SssI-treated DNA)/(total DNA molecules in SssI-treated DNA)].

\section{Quantitative reverse transcription PCR}

Complementary DNA was synthesized from $2 \mu \mathrm{g}$ of total RNA with use of Superscript III (Invitrogen). Real-time PCR was performed with SYBR ${ }^{\circledR}$ Green I and an iCycler thermal cycler (Bio-Rad Laboratories, Hercules, CA, USA). Standard samples with known numbers of template DNA molecules were prepared by serial dilution of a purified PCR product. The measured number of complementary DNA molecules was normalized to that of $G A D P H$. The primers and PCR conditions are given in Table S3.

\section{Survival curve and statistical analyses}

The Kaplan-Meier survival curves were drawn with SPSS 13.0J (SPSS Japan, Tokyo, Japan) for overall survival. The differences in the survival rates were evaluated by the Mantel-Cox test. The association between FAT4 methylation and clinicopathological characteristics was evaluated by the chi-square test. The presence of the CGI methylator phenotype (CIMP) was determined by the number of methylated genes in a cancer [5 or more of 12 genes (HAND1, PAX2, GREM1, LOX, HRASLS, CLDN3, THBD, ZNF177, FLNC, CTSL, F2R, and NT5E)], according to our previous report [26]. The difference in methylation levels in different groups was analyzed by the $t$ test. All of the analyses were performed with PASW Statistics version 18.0 (SPSS Japan, Tokyo, Japan), and a result was considered significant when $P<0.05$ was obtained by a twosided test.

\section{Results}

\section{Determination of the FAT4 transcription start site in gastric mucosae by $5^{\prime}$ RACE}

To evaluate the DNA methylation status of a promoter region, accurate determination of a transcription start site (TSS) is essential [27, 28]. The FAT4 TSS registered in the Database of Transcriptional Start Sites (DBTSS) was located approximately $1,500 \mathrm{bp}$ upstream of the TSS registered in the National Center for Biotechnology Information database (GRCh37.p10). Further, the TSS in the DBTSS was based not on expression data from the human stomach but on multiple other human adult tissues, such as the colon, heart, and kidney. The TSS in these tissues was consistent. Therefore, to determine a TSS of FAT4 in the stomach, we performed $5^{\prime}$ RACE, using RNA extracted from a normal gastric epithelial cell line, HGEC6B. We obtained a single RACE product of approximately 1,500 bp, and identified a single TSS (39/39 clones), which was consistent with the TSS of other tissues in the DBTSS (Fig. 1).

\section{Association between promoter methylation and loss of expression in GC cell lines}

We examined the association between FAT4 promoter methylation and its loss of expression in 13 human GC cell 
lines, the HGEC6B cell line, and normal gastric mucosae. Bisulfite sequencing of the FAT4 promoter region showed that it was highly methylated in two GC cell lines (AGS and GC2), moderately methylated in the KatoIII cell line, and unmethylated in the remaining $10 \mathrm{GC}$ cell lines, the HGEC6B cell line, and normal gastric mucosae (Figs. 2a, b, S1a). As shown by quantitative reverse transcription PCR, FAT4 was not expressed in the three GC cell lines with promoter methylation. In contrast, it was expressed in two (MKN7 and MKN45) of the ten GC cell lines with low or no methylation, in the HGEC6B cell line, and in normal gastric mucosae (Fig. 2c).

To confirm the effect of FAT4 promoter methylation on its expression, FAT4 methylation was removed with a demethylating agent, 5-aza-dC, in the two GC cell lines with the greatest methylation (AGS and GC2). FAT4 expression was induced in a dose-dependent manner with demethylation of its promoter (Figs. 2d, S1b). Addition of trichostatin A significantly enhanced FAT4 messenger RNA induction by 5-aza-dC in both GC cell lines. These data support the hypothesis that promoter methylation of FAT4 induces its loss of expression.

\section{Methylation and expression of FAT4 in primary GC specimens}

FAT4 promoter methylation in primary GC specimens was analyzed by qMSP of 82 GCs. FAT4 was methylated in 12 of the 82 GCs (14.6\%), with a cutoff value of $6 \%$ for the PMR, as previously reported [26, 29-31] (Fig. 3a). The presence of dense methylation of the promoter region was confirmed by bisulfite sequencing (Fig. 3b), and the fraction of densely methylated DNA molecules was consistent with the PMR values obtained by qMSP.

The correlation between promoter methylation and decreased expression was analyzed in $20 \mathrm{GC}$ specimens for which RNA was available. However, there was no significant difference between the mean FAT4 expression levels of five GCs with methylation and those of 15 GCs without methylation $(P=0.9328)$ (data not shown), most likely because surgical cancer specimens were contaminated with normal cells.

\section{Association between FAT4 methylation and clinicopathological characteristics of GCs}

We next analyzed the association between FAT4 promoter methylation and clinicopathological characteristics of GCs. FAT4 methylation was associated with the presence of the CIMP (Table 1). All of the 12 cancers with FAT4 methylation were CIMP positive, and 60 of 70 cancers without methylation $(86 \%)$ were CIMP negative $\left(P<10^{-9}\right)$. In contrast, FAT4 methylation was not associated with tumor
Fig. 2 Silencing of FAT4 by methylation of its promoter $\mathrm{CpG}$ island in gastric cancer (GC) cell lines. a FAT4 methylation levels analyzed by bisulfite sequencing of the $13 \mathrm{GC}$ cell lines, the HGEC6B cell line, and normal gastric mucosae. FAT4 was highly methylated in two GC cell lines (AGS and GC2) and moderately methylated in one GC cell line (KatoIII). b Representative data of bisulfite sequencing (AGS, GC2, MKN7, and HGEC6B cell lines). The genomic structure of the analyzed region is also shown. Arrow transcription start site, arrowheads positions of Uni-MSP primers, vertical lines individual CpG sites. The numbers of methylated $(M)$, unmethylated $(U)$, and intermediately methylated $(I)$ clones are shown. A molecule was considered methylated when more than 11 of the $13 \mathrm{CpG}$ sites were methylated and was considered unmethylated when fewer than two of the $13 \mathrm{CpG}$ sites were methylated. Black squares methylated $\mathrm{CpG}$ sites, white squares unmethylated $\mathrm{CpG}$ sites. c FAT4 expression levels in the 13 GC cell lines, the HGEC6B cell line, and normal gastric mucosae were analyzed by quantitative reverse transcription PCR. FAT4 was expressed in the HGEC6B cell line and normal gastric mucosae but was suppressed in most GC cell lines, except for MKN7 and MKN45. The lack of FAT4 expression in three cell lines (AGS, GC2, and KatoIII) supported its methylation-silencing. d FAT4 methylation and expression levels in the AGS and GC2 cell lines after 5-aza-2'-deoxycytidine (Aza) treatment. FAT4 methylation was removed by 5 -aza- $2^{\prime}$-deoxycytidine in both cell lines, and FAT4 expression was subsequently induced in a dose-dependent manner. Addition of trichostatin A (TSA) enhanced FAT4 induction by 5 -aza$2^{\prime}$-deoxycytidine

invasion, lymph node metastasis, or histological type. FAT4 methylation was also not associated with patient survival (Fig. 4).

\section{FAT4 methylation levels in noncancerous gastric mucosae with and without $H$. pylori infection}

FAT4 methylation in normal cells of the gastric mucosae was analyzed in 77 individuals (Fig. 5a). Specifically, the FAT4 methylation level was analyzed by qMSP in the gastric mucosae of healthy individuals (16 with and 17 without $H$. pylori infection) and noncancerous mucosae of GC patients (16 with and 28 without H. pylori infection). Among the healthy individuals, the PMR was significantly higher in the H. pylori-positive group than in the H. pylorinegative group (11.0 and 0.1, respectively; $P=0.00003$, $t$ test). Among the GC patients, the PMR tended to be higher in the H. pylori-positive group than in the H. pylorinegative group (4.1 and 1.9, respectively, $P=0.073, t$ test). Among the $H$. pylori-negative individuals, the PMR was significantly higher in the GC patients than in the healthy individuals (1.9 and 0.1 respectively; $P=0.006, t$ test), showing that the FAT4 methylation level in noncancerous gastric mucosa, as with the methylation levels of many other genes [5-7], was associated with GC risk in $H$. pylori-negative individuals.

GC risk is known to be associated also with the degree of gastric atrophy, and the association between the FAT4 methylation and GC risk was analyzed after stratification by the degree of gastric atrophy. The endoscopic degree of 
a

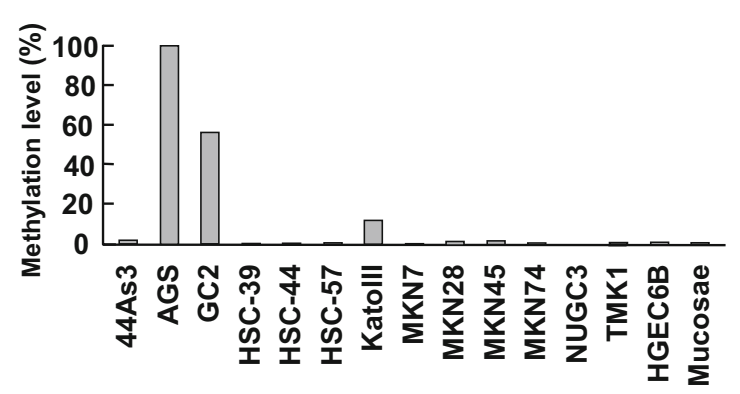

b
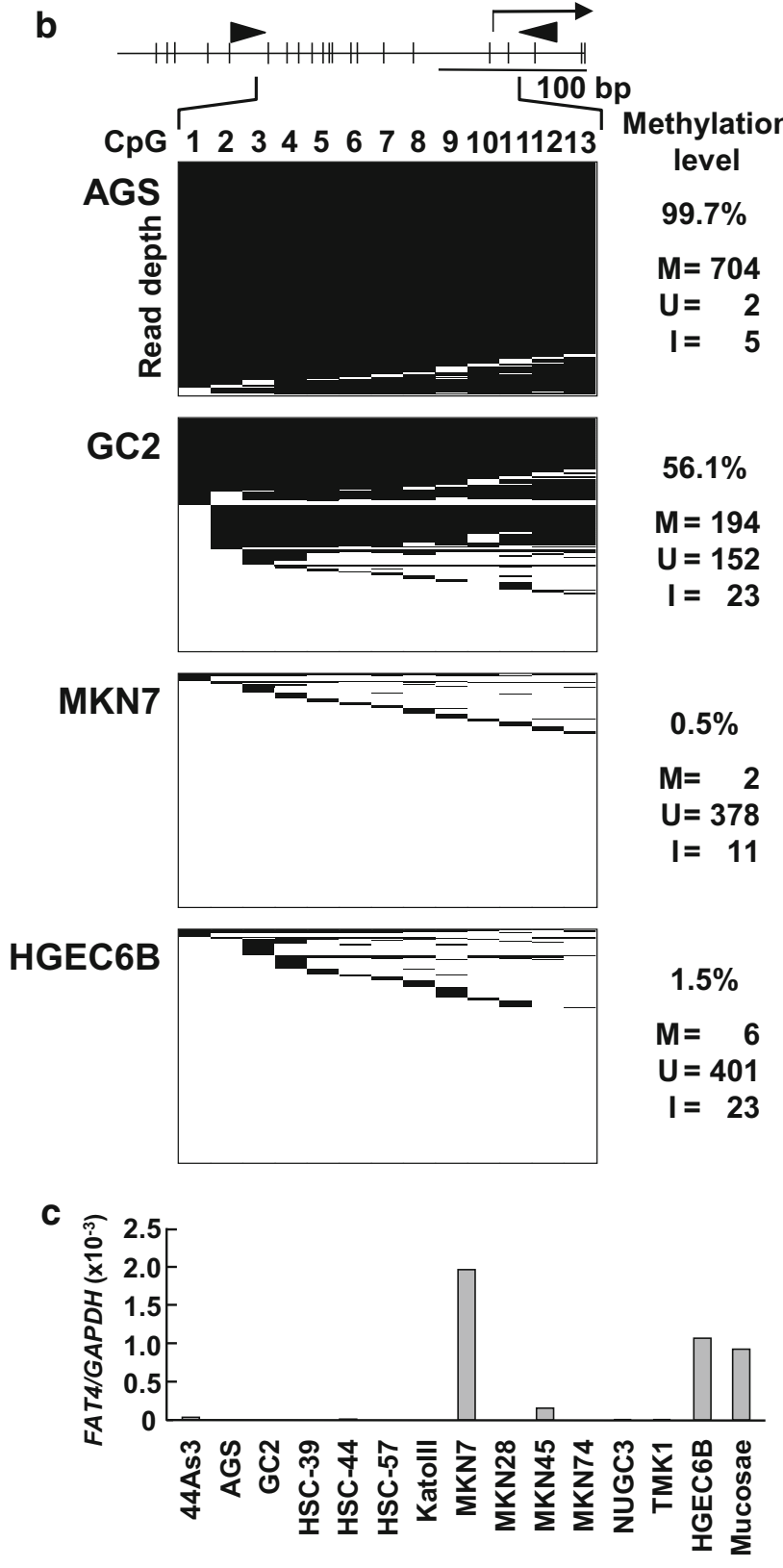

d
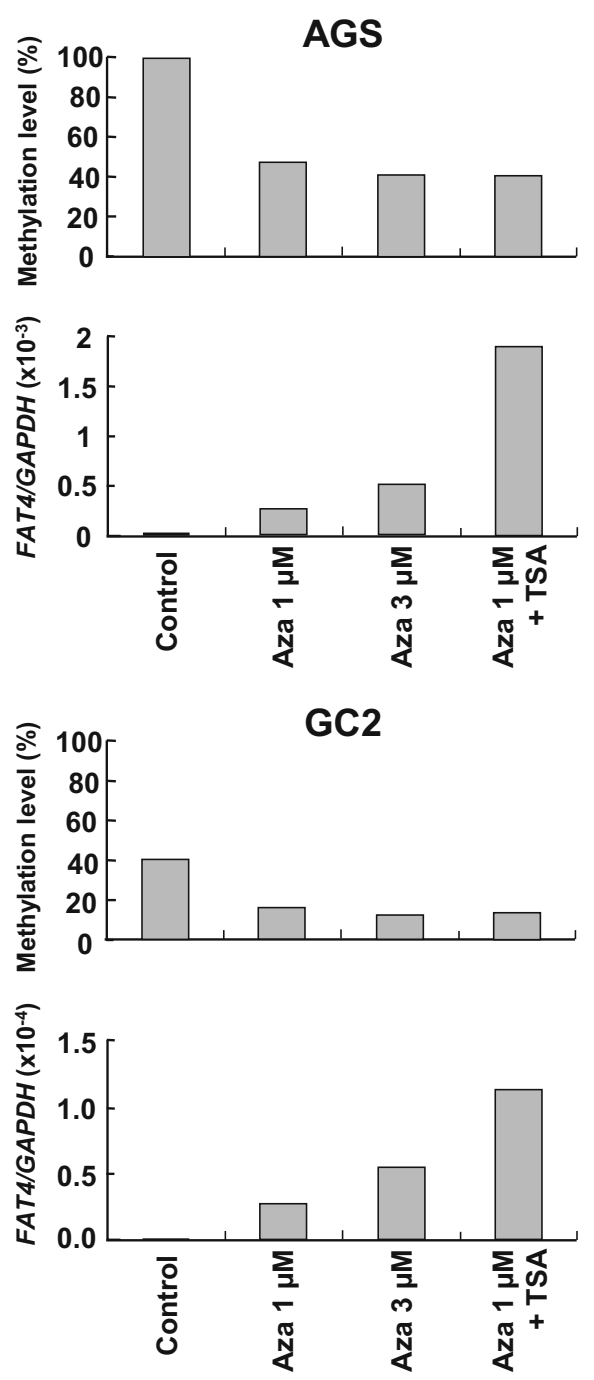
Fig. 3 FAT4 methylation in primary GC specimens obtained from $82 \mathrm{GC}$ patients who underwent gastrectomy. a Percentage of methylated reference $(P M R)$ obtained by quantitative methylationspecific PCR (qMSP) using primers specific to the methylated sequence (M-MSP primers) and primers common to methylated and unmethylated sequences (Uni-MSP primers). FAT4 was methylated in 12 of the 82 samples $(14.6 \%)$ with a cutoff value of $6 \%$. b The presence of dense methylation. Bisulfite sequencing was conducted in four GC specimens [two with methylation (GC \#1 and GC \#2) and two without $(G C \# 3$ and $G C \# 4$ ) by qMSP]. The region and $\mathrm{CpG}$ sites analyzed are shown in Fig. 2c. The fraction of densely methylated DNA molecules agreed with the PMR obtained by qMSP (noted below the sample number). Closed circle methylated $\mathrm{CpG}$ site, open circle unmethylated $\mathrm{CpG}$ site

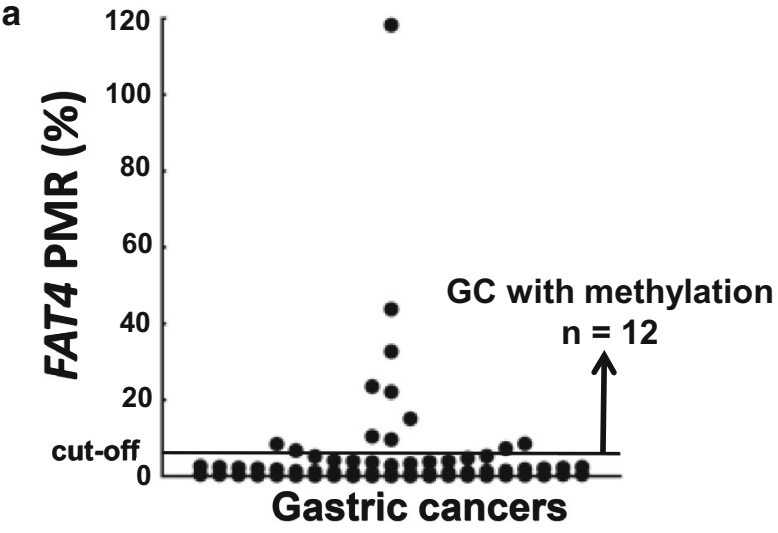

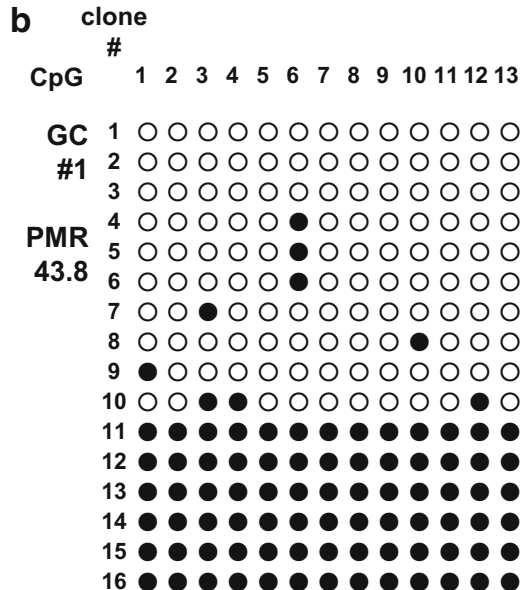

GC 10000000000000

\#2 2 \% ००००००००००००

$3000000 \bullet 000000$

$400 \bullet 0000000000$

PMR 5 O $०$ O O००००००००

$32.76 \bigcirc \bigcirc \bigcirc \bullet \bigcirc \bigcirc \bullet \bigcirc \bigcirc \bigcirc ० ० ०$

$7 \bullet \bigcirc \bullet \bullet \bigcirc \bigcirc \bigcirc \bullet \bullet \bigcirc ० ० ०$

$8 \bullet \bullet \bullet \bullet \bullet \bullet \bullet \bullet \bullet \bullet ० ० ०$

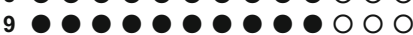

$10 \bullet \bullet \bullet \bullet \bullet \bullet \bullet \bullet \bullet \bullet \bigcirc \bigcirc ०$

$11 \bullet \bullet \bullet \bullet \bullet \bullet \bullet \bullet \bullet \bullet \bullet ० ०$

$12 \bullet \bullet \bullet \bullet \bullet \bullet ० ० \bullet \bullet$

$13 \bullet \bullet \bullet \bullet \bullet \bullet \bullet \bullet \bigcirc \bigcirc \bullet \bullet \bullet$

$14 \bullet \bullet \bullet \bullet \bullet \bullet \bullet \bullet ० \bullet \bullet$

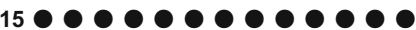

$16 \bullet \bullet \bullet \bullet \bullet \bullet \bullet \bullet \bullet \bullet \bullet \bullet \bullet ~$

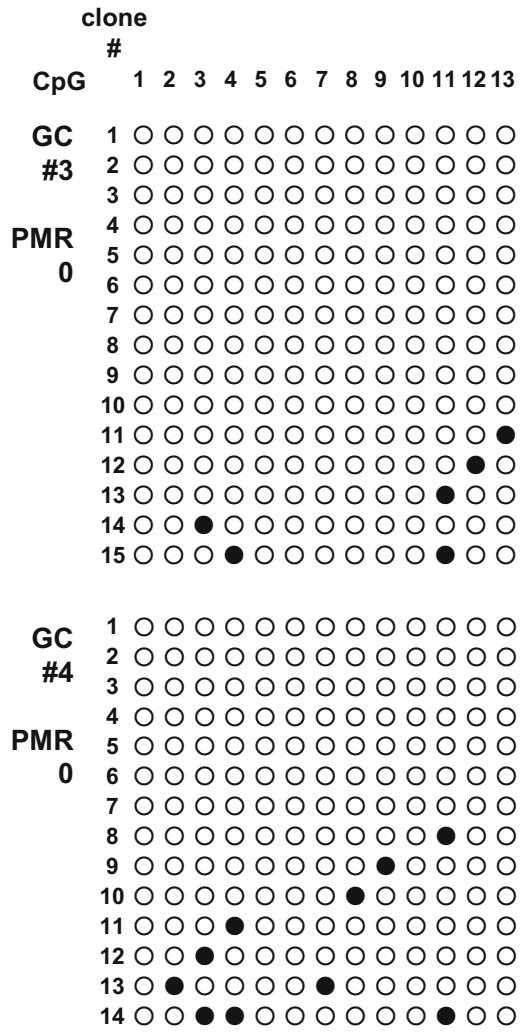

gastric atrophy was available for 60 of the 77 individuals. FAT4 methylation levels were higher in those with atrophy, but the trend was unclear (Fig. S2a). Because the presence of active $H$. pylori infection is known to induce temporary methylation that would decrease after eradication of $H$. pylori [5], the analysis was limited to the individuals without $H$. pylori infection, and the trend became clearer (Fig. S2b). Importantly, even among the individuals with gastric atrophy (and without active $H$. pylori infection), the FAT4 methylation level tended to be higher in GC patients (Fig. 5b).

\section{Discussion}

We showed that FAT4 was silenced by methylation of its promoter CGI in human GC. Because methylation of a CGI in a promoter region is known to be critical for gene silencing [27, 28], we identified a TSS used in the stomach, and confirmed the presence of a CGI in its directly upstream region. Methylation of the region was consistently associated with loss of expression in GC cell lines, and removal of FAT4 methylation by 5 -aza-dC induced its expression. Furthermore, in primary GCs, FAT4 was 
Table 1 Association between FAT4 promoter methylation and clinicopathological characteristics of gastric cancer patients

\begin{tabular}{lccc}
\hline Characteristics & \multicolumn{2}{l}{ FAT4 methylation } & $P^{\mathrm{a}}$ \\
\cline { 2 - 4 } & $\begin{array}{l}\text { Positive } \\
(n=12)\end{array}$ & $\begin{array}{l}\text { Negative } \\
(n=70)\end{array}$ & \\
\hline Tumor invasion & & 3 & 0.79 \\
T1 & 0 & 7 & \\
T2 & 2 & 21 & \\
T3 & 3 & 39 & 0.49 \\
T4 & 7 & & \\
Lymph node metastasis & 63 & 0.44 \\
Positive & 10 & 7 & \\
Negative & 2 & & \\
Histological type & & 58 & \\
Diffuse & 11 & 12 & \\
Intestinal & 1 & 10 & \\
CIMP & 12 & 60 & \\
Positive & 0 & & \\
Negative & & & \\
\hline
\end{tabular}

Eighty-two gastric cancer patients ( 50 male and 32 female patients; average age 55.6 years, age range 29-69 years) were analyzed. Gastric cancers were classified according to the Lauren classification system.

CIMP CpG island methylator phenotype

${ }^{\text {a }}$ Chi-square test

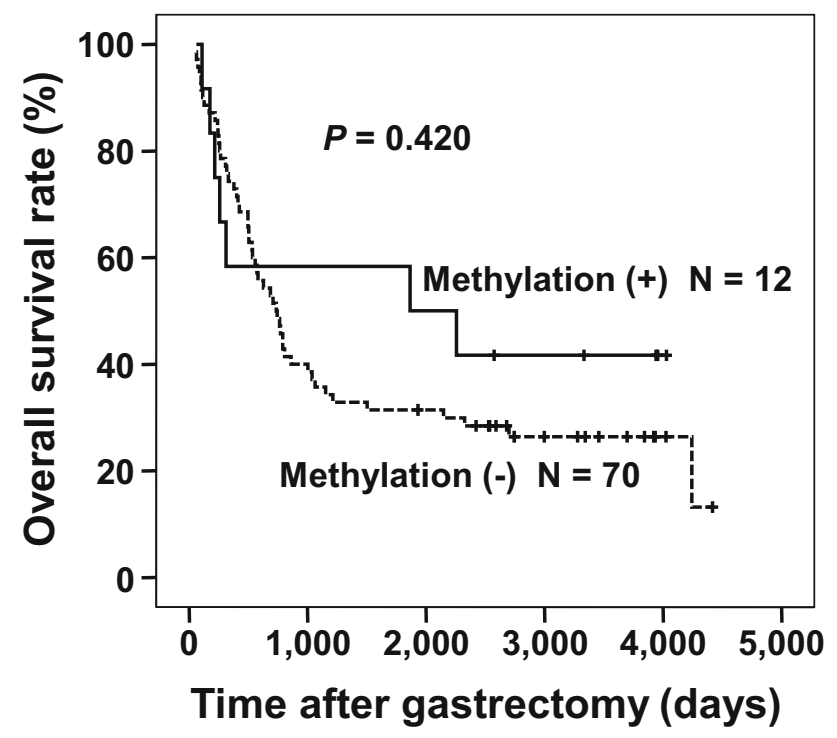

Fig. 4 Kaplan-Meier survival analysis of GC patients according to the FAT4 methylation status. FAT4 methylation was not associated with overall survival of GC patients

methylated in 12 of the 82 GCs $(14.6 \%)$. We then showed that methylation of the FAT4 promoter CGI was present not only in cancers but also in gastric mucosae, and the
Fig. 5 FAT4 methylation levels in noncancerous gastric mucosae. PMR values were analyzed by qMSP; a horizontal line represents the mean methylation level in each group. a The FAT4 methylation level in the gastric mucosae of healthy individuals and the noncancerous mucosae of GC patients with and without Helicobacter pylori infection. Among the healthy individuals, the PMR was significantly higher in those with $H$. pylori infection than in those without it. Among the GC patients, the PMR tended to be higher in the patients with $H$. pylori infection than in those without it. Among the individuals without $H$. pylori infection, the PMR was significantly higher in GC patients than in healthy individuals. b The FAT4 methylation level in noncancerous gastric mucosae without active H. pylori infection. The individuals were stratified by the existence of gastric atrophy, and, even among the individuals with gastric atrophy, the FAT4 methylation level was higher in GC patients

methylation level was associated with $H$. pylori infection (epigenetic field for cancerization), and, among individuals without $H$. pylori infection, with the presence of GC (namely, GC risk). Even limited to the individuals with gastric atrophy, the FAT4 methylation level tended to be higher in GC patients (Fig. 5b). To our knowledge, this is the first report on FAT4 methylation-silencing in GC and also on the presence of FAT4 methylation in noncancerous tissues of patients with any cancer. FAT4 can now be added to the list of driver genes that contribute to an epigenetic field, along with $C D H 1, C D K N 2 A, M L H 1$, and SWI/SNF chromatin remodeler genes $[3,16]$.

FAT4 methylation levels clearly increased in healthy individuals with $H$. pylori infection but only mildly increased among GC patients with $H$. pylori infection. The mild increase was considered to be due to (1) the already increased methylation levels in GC patients without H. pylori infection and (2) relatively weak chronic inflammation, a critical inducer of methylation $[8,9]$, in GC patients with $H$. pylori infection compared with healthy individuals with $H$. pylori infection. The association between high FAT4 methylation levels and the presence of a cancer was clear among the individuals without $H$. pylori infection, as with many other genes [5-7]. On the other hand, among the individuals with $H$. pylori infection, the healthy individuals had higher methylation levels than the GC patients. Again, a relatively weak inflammatory reaction in GC patients, compared with that in healthy individuals, was considered to be the reason for this.

FAT4 methylation was not associated with any clinicopathological characteristics except for the CIMP. It has been reported that even E-cadherin expression or inactivation was not associated with tumor invasion or metastasis. For example, Czyzewska et al. [32] observed no association between the expression of E-cadherin and invasion depth in GC. Chen et al. [33] found no association between E-cadherin and cancer stage, vascular invasion, lymph node involvement, or the presence of distant 


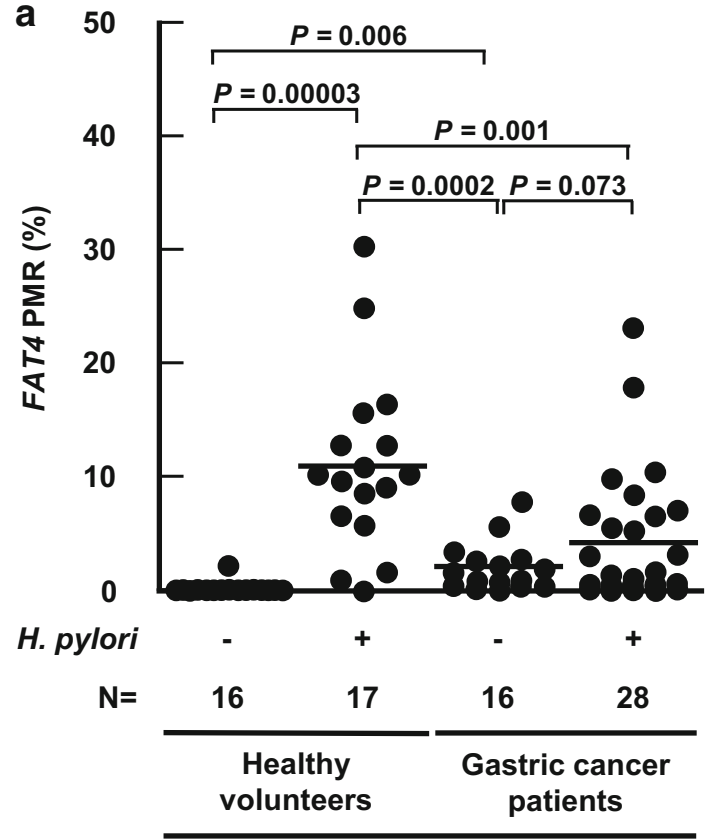

Gastric mucosae

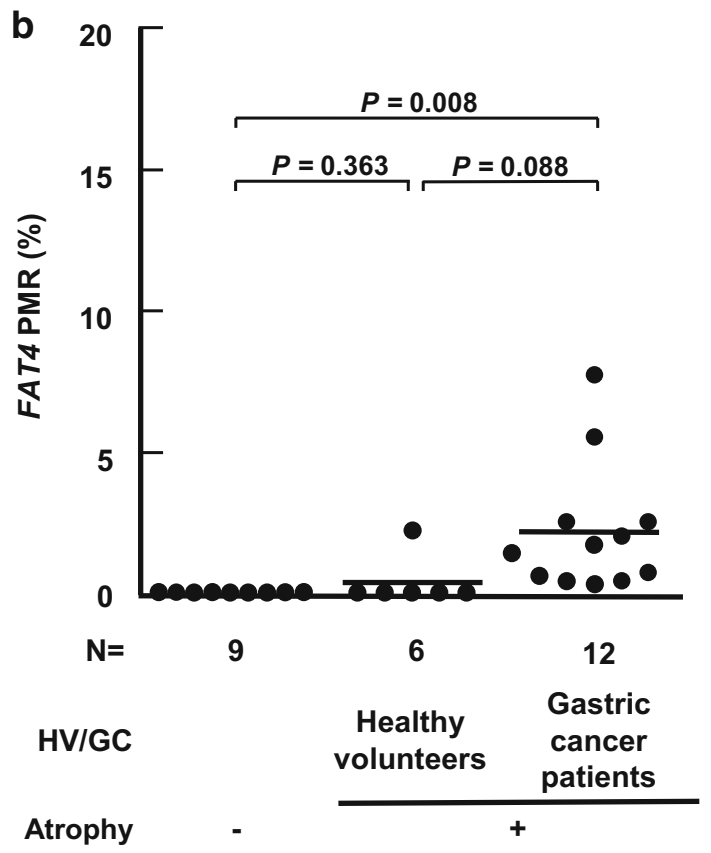

metastases. Mayer et al. [34] found no significant association between E-cadherin expression and tumor diameter, lymphatic vessel invasion, Borrmann classification, lymph node status, or manifest metastases. It appears possible that FAT4 methylation was not associated with these clinicopathological parameters. On the other hand, the CIMP in GCs is known to be associated with $M L H 1$ methylation and thus microsatellite instability [35-37] and with better prognosis [36, 37]. In this study, the number of GC cases with FAT4 methylation was relatively small (12 of 82 GC cases), which might have weakened the statistical power of survival analysis. With respect to histological types of GC, we initially expected that methylation of FAT4, a member of the cadherin superfamily, would be associated with the diffuse type, but there was no significant association. This could be explained if FAT4 silencing is involved in gastric carcinogenesis by Wnt activation rather than loss of cellular adhesion.

To distinguish GCs with FAT4 methylation, a PMR cutoff value of $6 \%$ was adopted, on the basis of previous reports [26, 29-31]. The use of a low cutoff value enables us to detect methylation in samples with low cancer cell counts but raises the concern of detecting methylation only in a fraction of cancer cells. The presence of heterogeneity among cancer cells makes it difficult to associate methylation status and gene expression levels. Indeed, in primary GCs, FAT4 expression was not different between GCs with and without FAT4 methylation. In addition to cancer cell heterogeneity, contamination by normal cells in GC specimens likely led to the ambiguous difference.

In conclusion, we showed that FAT4 was silenced by methylation of its promoter CGI in human GCs and that its methylation was associated with $H$. pylori infection and contributed to the development of an epigenetic cancerization field.

Acknowledgments The authors thank Drs. K. Asada, N. Hattori, and $\mathrm{H}$. Takeshima for their advice. The authors have declared that no competing interests exist. This work was supported by Practical Research for Innovative Cancer Control from Japan Agency for Medical Research and development, AMED (15ck0106023h0002); and the National Cancer Center Research and Development Fund (26A-15).

\section{Compliance with ethical standards}

Ethical standards All procedures followed were in accordance with the ethical standards of the responsible committee on human experimentation (institutional and national) and with the Helsinki Declaration of 1964 and later versions. Informed consent was obtained from all patients for the use of their samples.

Conflict of interest The authors declare that they have no conflict of interest.

\section{References}

Hattori N, Ushijima T. Compendium of aberrant DNA methylation and histone modifications in cancer. Biochem Biophys Res Commun. 2014;455:3-9.

Oue N, Sentani K, Sakamoto N, Yasui W. Clinicopathologic and molecular characteristics of gastric cancer showing gastric and intestinal mucin phenotype. Cancer Sci. 2015;106:951-8.

Ushijima T, Sasako M. Focus on gastric cancer. Cancer Cell. 2004:5:121-5. 
Yoda Y, Takeshima H, Niwa T, Kim JG, Ando T, Kushima R, et al. Integrated analysis of cancer-related pathways affected by genetic and epigenetic alterations in gastric cancer. Gastric Cancer. 2015;18:65-76.

Maekita T, Nakazawa K, Mihara M, Nakajima T, Yanaoka K, Iguchi $M$, et al. High levels of aberrant DNA methylation in Helicobacter pylori-infected gastric mucosae and its possible association with gastric cancer risk. Clin Cancer Res. 2006;12:989-95.

Kang GH, Lee S, Cho NY, Gandamihardja T, Long TI, Weisenberger DJ, et al. DNA methylation profiles of gastric carcinoma characterized by quantitative DNA methylation analysis. Lab Invest. 2008;88:161-70.

Shin CM, Kim N, Jung Y, Park JH, Kang GH, Kim JS, et al. Role of Helicobacter pylori infection in aberrant DNA methylation along multistep gastric carcinogenesis. Cancer Sci. 2010;101:1337-46.

Niwa T, Tsukamoto T, Toyoda T, Mori A, Tanaka H, Maekita T, et al. Inflammatory processes triggered by Helicobacter pylori infection cause aberrant DNA methylation in gastric epithelial cells. Cancer Res. 2010;70:1430-40.

Ushijima T, Hattori N. Molecular pathways: involvement of Helicobacter pylori-triggered inflammation in the formation of an epigenetic field defect, and its usefulness as cancer risk and exposure markers. Clin Cancer Res. 2012;18:923-9.

Nakajima T, Maekita T, Oda I, Gotoda T, Yamamoto S, Umemura S, et al. Higher methylation levels in gastric mucosae significantly correlate with higher risk of gastric cancers. Cancer Epidemiol Biomarkers Prev. 2006;15:2317-21.

Shin CM, Kim N, Park JH, Kang GH, Kim JS, Jung HC, et al. Prediction of the risk for gastric cancer using candidate methylation markers in the non-neoplastic gastric mucosae. J Pathol. 2012;226:654-65.

Ushijima T. Epigenetic field for cancerization. J Biochem Mol Biol. 2007;40:142-50.

Asada K, Nakajima T, Shimazu T, Yamamichi N, Maekita T, Yokoi $\mathrm{C}$, et al. Demonstration of the usefulness of epigenetic cancer risk prediction by a multicentre prospective cohort study. Gut. 2015;64:388-96

Nakajima T, Yamashita S, Maekita T, Niwa T, Nakazawa K, Ushijima $\mathrm{T}$. The presence of a methylation fingerprint of Helicobacter pylori infection in human gastric mucosae. Int $\mathbf{J}$ Cancer. 2009;124:905-10.

Takeshima H, Ushijima T. Methylation destiny: Moira takes account of histones and RNA polymerase II. Epigenetics. 2010;5:89-95.

Takeshima H, Niwa T, Takahashi T, Wakabayashi M, Yamashita S, Ando $\mathrm{T}$, et al. Frequent involvement of chromatin remodeler alterations in gastric field cancerization. Cancer Lett. 2015;357:328-38.

Zang ZJ, Cutcutache I, Poon SL, Zhang SL, McPherson JR, Tao J, et al. Exome sequencing of gastric adenocarcinoma identifies recurrent somatic mutations in cell adhesion and chromatin remodeling genes. Nat Genet. 2012;44:570-4.

Qi C, Zhu YT, Hu L, Zhu YJ. Identification of Fat4 as a candidate tumor suppressor gene in breast cancers. Int $\mathrm{J}$ Cancer. 2009;124:793-8.

Rauch TA, Wang Z, Wu X, Kernstine KH, Riggs AD, Pfeifer GP. DNA methylation biomarkers for lung cancer. Tumour Biol. 2012;33:287-96.

Katoh M. Function and cancer genomics of FAT family genes (review). Int J Oncol. 2012;41:1913-8.

Rock R, Schrauth S, Gessler M. Expression of mouse dchs1, fjx1, and fat-j suggests conservation of the planar cell polarity pathway identified in Drosophila. Dev Dyn. 2005;234:747-55.
Willecke M, Hamaratoglu F, Kango-Singh M, Udan R, Chen CL, Tao $\mathrm{C}$, et al. The Fat cadherin acts through the Hippo tumorsuppressor pathway to regulate tissue size. Curr Biol. 2006;16:2090-100.

Kimura K, Takemoto T. An endoscopic recognition of the atrophic border and its significance in chronic gastritis. Endoscopy. 1969;1:87-97.

Lauren P. The two histological main types of gastric carcinoma: diffuse and so-called intestinal-type carcinoma. An attempt at a histo-clinical classification. Acta Pathol Microbiol Scand. 1965;64:31-49.

Kaneda A, Kaminishi M, Sugimura T, Ushijima T. Decreased expression of the seven ARP2/3 complex genes in human gastric cancers. Cancer Lett. 2004;212:203-10.

Okochi-Takada E, Hattori N, Tsukamoto T, Miyamoto K, Ando T, Ito $\mathrm{S}$, et al. ANGPTL4 is a secreted tumor suppressor that inhibits angiogenesis. Oncogene. 2014;33:2273-8.

Li B, Carey M, Workman JL. The role of chromatin during transcription. Cell. 2007;128:707-19.

Lin JC, Jeong S, Liang G, Takai D, Fatemi M, Tsai YC, et al. Role of nucleosomal occupancy in the epigenetic silencing of the MLH1 CpG island. Cancer Cell. 2007;12:432-44.

Ota N, Kawakami K, Okuda T, Takehara A, Hiranuma C, Oyama K, et al. Prognostic significance of $\mathrm{p} 16^{\mathrm{INK} 4 \mathrm{a}}$ hypermethylation in non-small cell lung cancer is evident by quantitative DNA methylation analysis. Anticancer Res. 2006;26:3729-32.

Enomoto S, Maekita T, Tsukamoto T, Nakajima T, Nakazawa K, Tatematsu $\mathrm{M}$, et al. Lack of association between $\mathrm{CpG}$ island methylator phenotype in human gastric cancers and methylation in their background non-cancerous gastric mucosae. Cancer Sci. 2007;98:1853-61.

Ando T, Yoshida T, Enomoto S, Asada K, Tatematsu M, Ichinose M, et al. DNA methylation of microRNA genes in gastric mucosae of gastric cancer patients: its possible involvement in the formation of epigenetic field defect. Int $\mathrm{J}$ Cancer. 2009;124:2367-74.

Czyzewska J, Guzinska-Ustymowicz K, Ustymowicz M, Pryczynicz A, Kemona A. The expression of E-cadherin-catenin complex in patients with advanced gastric cancer: role in formation of metastasis. Folia Histochem Cytobiol. 2010;48:37-45.

Chen HC, Chu RY, Hsu PN, Hsu PI, Lu JY, Lai KH, et al. Loss of E-cadherin expression correlates with poor differentiation and invasion into adjacent organs in gastric adenocarcinomas. Cancer Lett. 2003;201:97-106.

Mayer B, Johnson JP, Leitl F, Jauch KW, Heiss MM, Schildberg FW, et al. E-cadherin expression in primary and metastatic gastric cancer: down-regulation correlates with cellular dedifferentiation and glandular disintegration. Cancer Res. 1993;53:1690-5.

Toyota M, Ahuja N, Suzuki H, Itoh F, Ohe-Toyota M, Imai K, et al. Aberrant methylation in gastric cancer associated with the $\mathrm{CpG}$ island methylator phenotype. Cancer Res. 1999;59:5438-42.

An C, Choi IS, Yao JC, Worah S, Xie K, Mansfield PF, et al. Prognostic significance of $\mathrm{CpG}$ island methylator phenotype and microsatellite instability in gastric carcinoma. Clin Cancer Res. 2005; 11:656-63.

Chang MS, Uozaki H, Chong JM, Ushiku T, Sakuma K, Ishikawa S, et al. CpG island methylation status in gastric carcinoma with and without infection of Epstein-Barr virus. Clin Cancer Res. 2006;12:2995-3002. 